\title{
Anxiety and Depressive Disorders in Adult Children Caring for Demented Parents
}

\author{
Jason R. Dura \\ Department of Psychiatry \\ Ohio State University College of Medicine \\ Janice K. Kiecolt-Glaser \\ Department of Psychiatry \\ Ohio State University College of Medicine
}

Karl W. Stukenberg

Ohio State University

\begin{abstract}
Current and lifetime rates of disorders outlined in the Diagnostic and Statistical Manual of Mental Disorders (3rd ed., rev., $D S M-I I I-R$ ) were compared in 78 adult children caring for a parent with a progressive dementia and 78 control subjects. The frequencies of depressive and anxiety disorders did not differ between groups in the years prior to caregiving, and there were no differences between groups in first-degree relatives' incidence of psychiatric disorder. During the years they had been providing care, $34 \%$ of caregivers met $D S M-I I I-R$ criteria: $24 \%$ for a depressive disorder and $10 \%$ for an anxiety disorder. In contrast, $8 \%$ of the matched controls met criteria during the same time period for depressive or anxiety disorders. Results support the chronic strains of caregiving being linked to the onset of both depressive and anxiety disorders in adult children caring for a demented parent.
\end{abstract}

There is general agreement that caregiving for a demented family member is associated with increased dysphoria, particularly for spouses (Light \& Lebowitz, 1989). For example, Dura, Stukenberg, and Kiecolt-Glaser (1990) found 30\% of spousal caregivers experienced a depressive disorder during the years they had been providing care. However, the association between caregiving and psychopathology in adult children caring for demented parents has not been studied as intensively. Researchers who have studied depression in mixed samples of dementia caregivers (i.e., spouse and adult children combined) have reported current disorder rates ranging from $14 \%$ to $47 \%$ of the samples (Gallagher, Rose, Rivera, Lovett, \& Thompson, 1989; Gallagher, Wrabetz, Lovett, Del Maestro, \& Rose, 1989; Morris, Morris, \& Britton, 1988). However, several differences between spousal and adult child caregivers argue for their separate analysis.

Children who care for parents are engaging in a role reversal that in itself has been discussed as a major developmental transition (Robinson \& Thurnher, 1979). The progression of care responsibility also typically follows a different pattern in adult children. Adult children are often assisting their nondemented parent, who is providing the bulk of care. In contrast to spousal caregivers, adult child caregivers generally do not share residence until the demented parent has lost the ability to live independently, or the spousal caregiver has become incapacitated or

This research was supported by Grant MH42096 from the National Institute of Mental Health to Janice K. Kiecolt-Glaser and Ronald Glaser and by funds from the Office of Geriatrics and Gerontology, Ohio State University College of Medicine.

Correspondence concerning this article should be addressed to Jason R. Dura, who is now at the Department of Psychology, Bowling Green State University, Bowling Green, Ohio 43403-0228. has died. Thus, although demands on time for adult child caregivers may be burdensome, they would not be at the level commonly experienced by spousal caregivers until residence is shared. In addition, although the loss of relationship quality is tragic for all, spousal caregivers would be expected to suffer more because of the primacy of their relationship to the demented. Generally, adult children should be more able to draw support from spouses and peers. However, this reasoning breaks down when residence is shared.

The arrival of a demented parent in the home of an adult child often coincides with multiple related stressors. Usually either the nondemented parent has died or become incapacitated, or the demented parent who had been living independently has deteriorated to such an extent that shared residence has been judged necessary for survival. Schedules must be altered, living arrangements adjusted, and routine rearranged. Everyone in the household will be affected and may share in care. Adult child caregivers often must attempt to balance care responsibilities among their demented parent, children, and work.

In examining the psychopathological consequences of caregiving for a demented parent, the current study differs from prior studies in several important ways: (a) Our sample was restricted to adult children caring for demented parents. (b) We examined subjects' lifetime history of disorders outlined in the Diagnostic and Statistical Manual of Mental Disorders (3rd ed., rev; $D S M-I I I-R)$, both prior to and during caregiving, as well as the incidence of disorder in their first-degree relatives; although personal and family history clearly mediate vulnerability (Depue \& Monroe, 1986), they have not been addressed in prior research on adult child caregivers. (c) Most studies have focused on only affective disorders. It is possible that caregiving is related to onset of a myriad of other Axis I psychiatric disorders 
(Popkin, Callies, Lentz, Colon, \& Sutherland, 1988), and the present study examines Axis I disorders comprehensively. (d) Prior studies addressing syndromal disorders have seldom used a comparison sample; instead, caregivers' data have been compared with epidemiological base rates. We used a sociodemographically matched control group. (e) In order to maximize the representativeness of our sample, our caregivers and controls were recruited widely from the community, and we assessed them both at our university clinic office and at home. Other data from our laboratory have shown that caregivers who are unable or unwilling to travel to a clinic site, despite the availability of free taxi service, are significantly more distressed than those who come for clinic assessment (Dura \& Kiecolt-Glaser, 1990).

We hypothesized that the stresses of caregiving for a demented parent would give rise to a greater cumulative incidence of psychiatric disorders during the caregiving period than would be found during the same time period in well-matched controls. Caregivers with a history of psychiatric disorder prior to caregiving were expected to be more vulnerable during caregiving than individuals without a past history, as were caregivers with a family history of psychiatric disorder. In addition, current dysphoria and depressive disorders were expected to be greater among caregivers than noncaregivers, though adult children were expected to have a lower cumulative incidence of depressive disorders than found in previous research with spousal caregivers. Also, adult children who shared residence with their parent were expected to be more dysphoric than those who lived separately.

\section{Method}

\section{Subjects}

Subjects were 78 adult children caring for a parent with progressive dementia and 78 control subjects. Each group included 12 men and 66 women, and groups were matched on gender, age, education, and income. Subjects were matched as pairs with control subjects recruited to match caregiving subjects. Age was matched \pm 3 years, and education was category matched using a \pm 1 -year category rule with categories as defined in Table 1 . Income was matched using $\$ 10,000$ increments with \pm 1 increment allowed. Matching criteria were chosen in an effort to allow comparison of peers, with caregiving being the critical variable differentiating groups. The specific matching variables were selected because of the role these variables may play in moderating risk for psychiatric disturbance.

Caregivers were recruited from three local dementia evaluation centers in area hospitals, neurologists' referrals, the city's Alzheimer's Disease and Related Disorders Association (ADRDA) support groups, the monthly ADRDA newsletter, respite care programs, and governmental caregiver support programs. Comparison subjects were recruited through newspaper advertisements and church groups; potential comparison subjects who reported any caregiving activities were excluded. The subjects were part of a larger study on chronic stress, immunity, and health. Subjects were excluded if they had major immunologically related health problems, such as cancer or recent surgeries. All subjects were paid $\$ 30$ for participation in the study.

\section{Psychiatric Diagnostic Data}

The Structured Clinical Interview--Nonpatient version (SCIDNP) is designed to enable a clinically trained interviewer to make reli-
Table 1

Characteristics of Caregivers and Comparison Subjects

\begin{tabular}{lcc}
\hline \multicolumn{1}{c}{ Characteristic } & Caregivers & Comparison subject \\
\hline Sex & & \\
Male & 12 & 12 \\
Female & 66 & 66 \\
Race & & \\
White & 72 & 71 \\
$\quad$ Black & 6 & 7 \\
Education & 5 & 4 \\
Partial high school or less & 17 & 20 \\
High school graduate & 24 & 28 \\
Partial college & 15 & 22 \\
College graduate & 17 & 13 \\
Graduate training & & 39 \\
Marital status & 44 & 20 \\
$\quad$ Married & 19 & 12 \\
$\quad$ Never married & 9 & 5 \\
Divorced & 6 & 2 \\
Widowed & 0 & \\
Separated & & \\
\hline
\end{tabular}

Note. Mean ages for caregivers and comparison subjects were 48.74 $(S D=10.11)$ and $51.62(S D=10.67)$, respectively.

able and valid $D S M-I I I-R$ diagnoses (Riskind, Beck, Berchick, Brown, \& Steer, 1987; Spitzer, Williams, Endicott, \& Gibbon, 1987). The SCID-NP has adequate to superior validity and reliability (Riskind et al., 1987; Spitzer et al., 1987) with greatest precision in the diagnosis of current disorder. Interviews were done by advanced clinical psychology graduate students or by a clinical psychologist. The diagnostic interviews provided current and lifetime incidence of affective disorders with the approximate date (or dates) of onset noted.

As suggested by Becker and Morrissey (1988) and Gallagher, Rose et al. (1989), interviewers were careful to attribute symptoms to depression only when their presence or intensity was not explicable simply on the basis of the caregiving situation itself or related to normal changes associated with aging. For example, in evaluating changes in slcep, interviewers inquired about sleep quality compared with that of the last few years, not current sleep quality compared with that at a much earlier time; similarly. if a dementia patient had a disrupted diurnal wake-sleep cycle that interfered with the caregiver's sleep, this was not considered a sleep disturbance in the caregiver.

Interrater reliability for SCID-NP diagnoses was calculated using randomly selected audiotaped interviews for $34(22 \%)$ of the subjects. The kappa coefficient was .93 for depressive disorders across categories and demonstrates adequate interrater reliability. It is important to note that kappa is affected by base rates that in the current sample, were quite low. In addition, interviewers were not unaware as to whether subjects were caregiving.

Psychopathology in first-degree relatives was assessed using Family History Research Diagnostic Criteria (FH-RDC; Andreasen, Endicott, Spitzer, \& Winokur, 1977), a method that has good to excellent reliability for specific FH-RDC disorders but not for the residual category of other psychiatric disorders (Zimmerman, Coryell, Pfohl, \& Stangl, 1988). The FH-RDC is the best standardized method for assessing family history of psychiatric disturbance available, but other family members' symptom reports can be selective, and data should be interpreted cautiously.

The FH-RDC interview followed the SCID-NP and used hierarchical probes to assess history in parents, siblings, and children across 12 categories. The categories were chronic schizophrenia, schizo-affective disorder, single episode of depressive disorder, recurrent unipolar de- 
pression, single episode or recurrent manic disorder, bipolar disorder, senile organic brain syndrome, unspecified functional psychosis, alcoholism, drug use disorder, antisocial personality, or other psychiatric disorder. Senile organic brain syndrome was not assessed in this study because a disproportionate finding of this condition in caregivers' parents follows from group selection (i.e., caring for a parent with progressive dementia).

For caregivers, onset of caregiving was obtained from a separate questionnaire so that a determination could be made as to whether the disorder (or disorders) began during caregiving. The caregiving date of onset was determined by reviewing need for assistance from the point of the first neurological evaluation backward in months. Caregivers were required to provide examples of specific instances of providing assistance for caregiving to be scored as ongoing. Total caregiving time was then determined by the length of time since assistance began. For control subjects, the onset date of caregiving for their matched caregiver was used for comparison purposes; for example, if the caregiver had been providing care for 5 years, we examined the matched control subject's incidence of affective disorder during the last 5 years.

\section{Depression Scales}

The Hamilton Depression Rating Scale (HDRS), a 24-item, interviewer-rated measure of depression, provided information on depressive symptomatology for the week prior to the interview period (Hamilton, 1967). Interrater reliability was $.84(d f=15)$ for the HDRS, calculated for $10 \%$ of the subjects using audiotapes.

The short form of the Beck Depression Inventory (BDI), a 13-item subset of the original instrument (Beck \& Beck, 1972), was used. Each item presents four sentences and the subjects endorse the sentence or sentences that best describe the way they have been feeling in the past week.

\section{Dementia Assessment}

Other research has demonstrated that family members' ratings provide one source of reliable data on dementia patients' functioning. For example, in a sample of 82 elderly patients, the majority of whom had a differential aptitude test (DAT) diagnosis, there was a high degree of concordance between family and staff members' ratings regarding 10 areas of patient functioning (Reifler, Cox, \& Hanley, 1981).

The Blessed Dementia Scale (BDS; Blessed, Tomlinson, \& Roth, 1968) was developed to measure impairments in a demented person's abilities across daily living, self-care, and personality domains. Higher scores on this 22-item scale denote greater decrements in ability, with a potential range of 0 to 28 . BDS scores correlate with senile plaque count during postmortem histological examination as well as impairments in neuropsychological testing and can be used to differentiate degree of dementia (Blessed et al., 1968; Erkinjuntti, Hokkanen, Sulkava, \& Palo, 1988).

The Memory and Behavior Problem Checklist (MBPC; Zarit, Reever, \& Bach-Peterson, 1980) measures behavioral excesses and deficits in dementia patients and the caregiver's reaction to those problems. The 29 items include some of the problems most distressing to caregivers; for example, the patient's inability to dress and feed himself or herself, incontinence, inability to communicate, tendency to hide things, and inability to recognize familiar people. The scalc provides three scores: a measure of the frequency of problem behaviors, a measure of associated caregiver distress, and a score that combines frequency and reaction to produce a summary measure. Factor analytic research with the MBPC has shown three discrete domains: need for assistance with self-care and instrumental activities of daily living; memory problems and psychiatric symptoms; and communication difficulties (Dura, Bornstein, \& Kiecolt-Glaser, 1990). The MBPC measures current symptoms and associated subjective caregiver distress, not impairment of the patient per se, as many behavior problems are most prevalent in the middle stages of dementia (Haley, Levine, Brown, \& Bartolucci, 1987).

The Global Deterioration Scale (GDS; Reisberg, Ferris, DeLeon, \& Crook, 1982) provides a dementia stage rating in patients who have been previously diagnosed with dementia. The scale has well-described anchor points, from 1 (no cognitive decline) to 7 (very severe cognitive decline). Ratings were based on caregivers' reports of the affected family members' history and current functioning. Interrater reliability using the GDS was assessed by comparing ratings made by the original interviewer with those of a clinical psychologist with geriatric assessment experience. The psychologist, unaware of the prior rating, based his ratings on information from the BDS and MBPC. Reliability was computed for 22 randomly chosen cases ( $28 \%$ ); the Pearson product-moment correlation between the original and comparison GDS scores was $.78(d f=22)$.

\section{Results}

\section{Subject Characteristics}

Demographic characteristics are presented by group in Table 1. The matching procedures were successful in producing groups that did not differ on age, education, or income. The modal subject reported an annual family income between $\$ 30,000$ and $\$ 40,000$. Although we did not match comparison subjects to caregivers exactly on marital status, the inclusion of more divorced or separated control subjects actually worked against confirmation of the experimental hypotheses because divorce and separation are associated with higher rates of psychopathology (Kessler, Price, \& Wortman, 1985).

Thirty-two caregivers cared for their parent in their own home, 21 cared for a parent living in another family member's home or living alone, and 25 of the patients were institutionalized. Caregivers assisting a parent living alone assisted a mean $3.19 \mathrm{hr}(S D=3.08)$ per day and had been providing assistance a mean 30.62 months $(S D=22.17)$. Caregivers sharing residence assisted a mean $7.93 \mathrm{hr}(S D=5.77)$ per day and had been providing care a mean 41.39 months $(S D=33.29)$. Caregivers assisting a parent in a nursing home assisted a mean $1.55 \mathrm{hr}$ per day $(S D=1.08)$ and had been providing assistance a mean 67.40 months $(S D=51.38)$.

The majority of the caregivers' parents were severely demented. Only $19 \%$ of subjects fell within Stages 2 through 4 on the GDS, with 5\% in Stage 2 (forgetfulness), $1 \%$ in Stage 3 (early confusional), and $13 \%$ in Stage 4 (late confusional). In contrast, the majority clearly fell in later stages, with $30 \%$ in Stage 5 (early dementia), 33\% in Stage 6 (middle dementia), and $18 \%$ in Stage 7 (late dementia). Sixty-three of the caregivers' parents had a DAT diagnosis, 3 were diagnosed with multi-infarct dementia, 6 with Parkinson's disease, 3 with Huntington's disease, and 3 with an unspecified dementia; $48 \%$ had received their diagnosis at one of three local neurology centers that used the National Institute of Neurological and Communicative Disorders and Stroke/Alzheimer's Disease and Related Disorders Association workgroup standards for probable DAT (McKhann et al., 1984).

\section{Past and Current Depressive Disorders}

Prior to caregiving, 23 caregivers (30\%) and 15 comparison (19\%) subjects reported a depressive disorder, a nonsignificant 
difference, $\chi^{2}(1, N=156)=2.23$. Most of these were cases of major depression. Six caregivers and 2 comparison subjects had previously met criteria for dysthymia, and 1 comparison subject met criteria for depressive disorder not otherwise specified (NOS). No comparison subjects had a current case of depressive disorder, whereas 14 caregivers $(18 \%)$ had current depressive disorders, $\chi^{2}(1, N=156)=15.38, p<.01$.

During the caregiving period, caregivers reported significantly more depressive disorders than did comparison subjects -19 of $78(26 \%)$ versus 3 of $78(4 \%), \chi^{2}(1, N=156)=13.55, p<$ .01 - with diagnoses shown in Table 2 . Seven of the 20 caregivers who reported a depressive episode during the caregiving period reported a prior depressive episode; six of these prior episodes were major depression, whereas one was dysthymia.

Seven of 23 caregivers with a prior depressive diagnosis (37\%) experienced symptoms meriting a depressive disorder diagnosis while caregiving. Twelve of 55 caregivers with no prior depressive disorder diagnosis (22\%) received a depressive disorder diagnosis during caregiving. Though caregivers with a prior depressive diagnosis appeared more likely to have a depressive disorder while caregiving, the difference was not statistically significant, $\chi^{2}(1, N=78)=.65$.

During the matched caregiving time periods, caregivers reported more cases of major depressive disorder than comparison subjects: 13 of $78(18 \%)$ versus 3 of $78(4 \%), \chi^{2}(1, N=156)=$ $6.96, p<.01$. Caregivers did not report significantly more cases of dysthymia, nor did they report more cases of depressive disorder NOS.

Depressive diagnosis did not vary consistently by gender. Among caregivers, 3 of 12 men (25\%) and 16 of 66 women (24\%) reported a depressive episode while caregiving, whereas 4 of 12 men $(33 \%)$ and 19 of 66 women $(29 \%)$ reported one prior to caregiving. Among comparison subjects, 1 of 12 men (8\%) and

Table 2

Frequency of Depressive Disorders by Group

\begin{tabular}{lcc}
\hline Depressive disorder & Caregiver & Comparison subject \\
\hline Current* & & \\
Major depressive disorder & 8 & 0 \\
Dysthymic disorder & 4 & 0 \\
Depressive disorder Nos & 2 & 0 \\
During caregiving years* & & \\
$\quad$ (cumulative) & 13 & 3 \\
Major depressive disorder* & 4 & 0 \\
Dysthymic disorder & 2 & 0 \\
Depressive disorder NOS & & \\
Before caregiving years & 17 & 12 \\
$\quad$ (cumulative) & 6 & 2 \\
Major depressive disorder & 0 & 1 \\
Dysthymic disorder & & \\
Depressive disorder NOS & & \\
\hline
\end{tabular}

Note. For comparison subjects, the caregiving onset date for their matched caregiver was used for comparison purposes; for example, if the caregiver had been providing care for 5 years, we examined the noncaregiver's incidence of depressive disorders during the last 5 years. The $n s=78$ for both carcgivers and comparison subjects. NOS = not otherwise specified.

$* p<.01$.
Table 3

Frequency of Anxiety Disorders by Group

\begin{tabular}{lcc}
\multicolumn{1}{c}{ Anxiety disorder } & Caregiver & Comparison subject \\
\hline Current & & \\
Panic disorder & 0 & 0 \\
Social phobia & 1 & 0 \\
$\quad$ Generalized anxiety disorder & 5 & 0 \\
During caregiving years* & & \\
$\quad$ (cumulative) & 1 & 0 \\
Panic disorder & 1 & 0 \\
Social phobia & 6 & 1 \\
Generalized anxiety disorder & & \\
Before caregiving years & & \\
$\quad$ (cumulative) & 2 & 3 \\
Panic disorder & 2 & 0 \\
Social phobia & 2 & 1 \\
Simple phobia & 3 & \\
Generalized anxiety disorder & 3 & \\
\hline
\end{tabular}

Note. For comparison subjects, the caregiving onset date for their matched caregiver was used for comparison purposes; for example, if the caregiver had been providing care for 5 years, we examined the noncaregiver's incidence of anxiety disorders during the last 5 years. Both for caregivers and comparison subjects, $n=78$.

$* p<.05$.

17 of 66 women (26\%) reported a depressive disorder either prior to or during the matched caregiving time period.

Among caregivers, residence of their demented parent was not reliably related to prevalence of depressive disorders during caregiving. Six caregivers who shared residence, 6 who cared for a parent who lived with another family member or alone, and 7 who continued to provide care for a parent residing in a longterm care facility had a depressive diagnosis during caregiving.

\section{Anxiety Disorders}

Caregivers received more diagnoses of anxiety disorders during caregiving, though there were no significant differences before caregiving (see Table 3). During caregiving, 6 caregivers met criteria for generalized anxiety disorder, 1 met criteria for panic disorder, and 1 met criteria for phobic disorder. In contrast, only 1 comparison subject was diagnosed with a generalized anxiety disorder. Thus, 8 of 78 caregivers (10\%) had an anxiety disorder, whereas only 1 of 78 comparison subjects had an anxiety disorder $(1 \%), \chi^{2}(1, N=156)=5.78, p<.05$. (Two caregivers with anxiety disorders had depressive disorders concurrently.)

Prior to caregiving, 3 caregivers met generalized anxiety disorder criteria, 2 met criteria for panic disorder, and 4 met criteria for various phobias. Among comparison subjects, 1 met criteria for generalized anxiety disorder, 3 met criteria for panic disorder, and 2 met criteria for phobic disorders.

\section{Other Axis I Disorders}

Other Axis I disorders were infrequent among both caregivers and comparison subjects. During caregiving, 2 caregivers 
met criteria for adjustment disorder. One comparison subject met criteria for alcohol abuse, and 2 met criteria for adjustment disorder.

Prior to caregiving, 5 caregivers had met criteria for adjustment disorder, 2 had met criteria for substance abuse disorder, and 1 had met criteria for a psychotic disorder. Among comparison subjects, 3 had met criteria for adjustment disorder, 5 had met criteria for substance abuse disorders, and 1 had met criteria for a psychotic disorder.

\section{Family History}

Four of the 19 caregivers with depressive disorders during the years they were caregiving had a family history of mental illness: 1 caregiver with major depression had an alcoholic parent, 2 dysthymic caregivers had siblings with affective disorders, and 1 caregiver with depressive disorder NOS had a sibling with an unspecified psychiatric disorder. None of the 3 comparison subjects who had depressive disorders during their matched caregiving time period had a family history of psychiatric disorder. Four of the 17 caregivers with major depressive disorder prior to caregiving reported a family history of mental illness, as did 3 control subjects. Thus, family history did not appear strongly associated with major depression in comparison subjects or caregivers.

\section{Vulnerability and Sociodemographic Variables}

Greater vulnerability to depressive disorders has been associated with lower education and lower income across a number of studies (Kessler et al., 1985; Schulz, Tompkins, \& Rau, 1988). Caregivers who had met $D S M-I I I-R$ criteria during caregiving had lower current income, averaging between $\$ 10,000$ and $\$ 19,000$, in comparison with an average income of between $\$ 20,000$ and $\$ 29,999$ for those not meeting criteria, $F(1,73)=$ $8.18, p<.006$. (Three caregivers declined to report income.) Caregivers who had met $D S M-I I I-R$ criteria during caregiving were comparable in education and age $\left(F_{\mathrm{S}}<1\right)$.

Caregivers with children at home and caregivers without children had comparable rates of $D S M-I I I-R$ diagnoses. Eighteen of 56 caregivers with children (32\%) and 7 of 22 caregivers without children $(32 \%)$ had either depressive or anxiety disorders while caregiving, $\chi^{2}(1, N=78)=p<.001$.

Caregivers with intact marriages were compared with caregivers who were single, divorced, or widowed. Twelve of 48 married caregivers (25\%) received diagnoses in comparison with 13 of 30 nonmarried caregivers $(43 \%), \chi^{2}(1, N=78)=2.85$, $p<.09$. Although it did not reach statistical significance, this finding bears testing in a larger sample.

The majority of caregivers combined caregiving with employment. Employment had no discernable effect on diagnosis. Seventeen of 52 employed caregivers (33\%) received depressive or anxiety disorder diagnoses in comparison with 8 of 25 unemployed caregivers (32\%). (One caregiver who did not receive a diagnosis did not provide employment information.)

\section{Interviewer- and Self-Rated Depressive Symptoms}

Caregivers' current mean interviewer-rated depression on the HDRS $(M=7.37, S D=6.19)$ was significantly higher than that found in the comparison subjects $(M=2.66, S D=3.15), F(1$, $151)=34.44, p<.0001$. Analyses of variance (ANOVAs) for depressive symptoms included gender as a variable because women typically report more depressive symptoms than men (Kessler et al., 1985). Women's mean level of depression on the HDRS was comparable to that of the men, $F(1,151)=1.02$. There was no significant Group $\times$ Gender interaction $(F<1)$. Similarly, caregivers' mean self-rated depression $(M=6.02$, $S D=6.34$ ) was significantly higher than that of controls on the BDI $(M=2.78, S D=3.77), F(1,114)=6.90, p<.009$. Again, there was no significant gender effect $(F<1)$ or interaction, $F(1$, 114 ) $=1.90$. (The BDI was added after the study's inception and was not available for all subjects.)

Most caregiving studies have not found reliable relationships between depression in caregivers and the extent of patient impairment (Haley et al., 1987; Quayhagen \& Quayhagen, 1988). In this study, the correlation between HDRS ratings and GDS stage was $-.04,(d f=77)$. The correlation between the HDRS and years spent caregiving was $.02(d f=77)$, whereas average hours spent per day in caregiving correlated, $r(77)=.15$, with the HDRS.

As noted earlier, Cohen and Eisdorfer (1988) found significantly greater depression in at-home caregivers. When distress as measured by the HDRS was compared, there was no difference between caregivers sharing residence $(n=32 ; F<1)$, providing care to a demented parent living alone or with another relative ( $n=21 ; F<1$ ), or continuing care for a parent placed in a long-term care facility $(n=25 ; F<1)$.

Cohen and Eisdorfer (1988) excluded any caregiver from their study who was currently in a support group, whereas Gallagher, Wrabetz, et al. (1989) used subjects who had sought treatment in a psychoeducational program for caregiving-related distress. In order to assess the possibility that those subjects in our study who were recruited from support groups differed from those who were not, we compared depression scores for the two groups. Support group members and nonmembers were comparable on HDRS scores, $F(1,75)=1.07$.

\section{Discussion}

Adult children assisting a demented parent experienced significantly more depressive and anxiety disorders during the years they provided care than matched controls experienced during the same time period. In contrast, adult child caregivers and paired comparison subjects had comparable rates of depressive and anxiety disorders prior to the caregiving period. Caregivers with neither a family history nor a personal history of psychiatric disorder developed depressive and anxiety disorders while caregiving. These data suggest that the chronic strains of caregiving are related to the onset of depressive and anxiety disorders in adult children providing assistance to their demented parents.

Contrary to our original hypothesis, adult child caregivers experienced depressive disorders at rates comparable to spousal caregivers (i.e., $26 \%$ of adult child caregivers in the current sam- 
ple vs. $30 \%$ of spousal caregivers in prior research; Dura, Stukenberg et al., 1990). The present data indicated that $22 \%$ of adult child caregivers who had not previously met criteria experienced symptoms consistent with a depressive disorder at some point during caregiving. Among adult children with a prior depressive disorder diagnosis, approximately $37 \%$ again experienced a depressive disorder while caregiving. This difference in vulnerability was not statistically significant. Whether a larger sample size would have produced statistical significance remains to be determined by future research.

Although current diagnostic reliability data were adequate, the present findings should be interpreted cautiously. Diagnostic reliability may be inflated by low base rates that were present in the current study. Also, the comparison diagnostician, whose rating provided for calculation of reliability, was unaware of prior diagnostic findings but not of caregiving status. It should be noted that our rates for depressive disorders among caregivers are lower than those reported in most other caregiver studies using structured psychiatric interviews (Gallagher, Rose et al., 1989; Gallagher, Wrabetz et al., 1989).

Rates of $D S M-I I I-R$ diagnosis did not vary by residence of the demented parent, support group membership, caregiver employment, caregiver gender, presence or absence of children in the caregivers' homes, or marital status. In contrast, lower income was associated with greater likelihood of developing a depressive disorder. This finding is consistent with a large literature that associates depressive vulnerability with lower socioeconomic status (Depue \& Monroe, 1986; Kessler et al., 1985). Income serves as a buffer against the negative effects of stress generally, and the present data support this relationship for the chronic strains of caregiving as well.

The finding that adult child caregivers had more anxiety disorders than comparison subjects contrasts with similar research using spousal caregivers (Dura, Stukenberg, et al., 1990). This finding was unexpected and not predicted. The present data argue against a specific link between caregiving and depressive disorders in adult children caregivers. Six of 8 caregivers with anxiety disorders represented unique cases in that they did not have depressive disorder, the most frequent concurrent diagnosis. Recently, calls have been made for caregiving researchers to explore anxiety as a response to caregiving stress (Gallagher, Wrabetz et al., 1989). The present data support this as a potential research direction for study of adult children who assist a demented parent.

As predicted, current self- and other-rated dysphoria was higher in caregivers than in comparison subjects. Contrary to studies among noncaregivers, no gender effects were found in reports or ratings of current dysphoria in caregivers. That is, female and male caregivers had comparable rated and self-reported dysphoria.

The current findings argue strongly for additional research regarding caregiving and vulnerability to diagnosable depression and anxiety in adult children caring for demented parents. Caregiving, regardless of a variety of situational variables, appears to accrue significant mental health risks. Greater understanding of the mechanisms through which caregiving promotes mental illness is critical, especially as our population ages and caregiving becomes normative.

\section{References}

Andreasen, N. C., Endicott, J., Spitzer, R. L., \& Winokur, G. (1977). The family history method using diagnostic criteria. Archives of General Psychiatry, 34, 1229-1235.

Beck, A. T., \& Beck, R. W. (1972). Screening depressed patients in family practice: A rapid technique. Postgraduate Medicine, 81-85.

Becker, J., \& Morrissey, E. (1988). Difficulties in assessing depressivelike reactions to chronic severe external stress as exemplified by spouse caregivers of Alzheimer patients. Psychology and Aging, 3, 300-306.

Blessed, G., Tomlinson, B. E., \& Roth, M. (1968). The association between quantitative measures of dementia and senile change in the cerebral grey matter of elderly subjects. British Journal of Psychiatry, $114,797-811$.

Cohen, D., \& Eisdorfer, C. (1988). Depression in family members caring for a relative with Alzheimer's disease. Journal of the American Geriatrics Society, 36, 885-889.

Depue, R. A., \& Monroe, S. M. (1986). Conceptualization and measurement of human disorder in life stress research: The problem of chronic disturbance. Psychological Bulletin, 99, 36-51.

Dura, J. R., Bornstein, R. A., \& Kiecolt-Glaser, J. K. (1990). Refinements in the assessment of dementia-related behaviors: Factor analysis of the memory and behavior problem checklist. Psychological Assessment: A Journal of Consulting and Clinical Psychology, 2, 129133.

Dura, J. R., \& Kiecolt-Glaser, J. K. (1990). Sample bias in caregiving research. Journal of Gerontology, 45, 200-204.

Dura, J. R., Stukenberg, K. W., \& Kiecolt-Glaser, J. K. (1990). Chronic stress and depressive disorders in older adults. Journal of Abnormal Psychology, 99, 284-290.

Erkinjuntti, T., Hokkanen, L., Sulkava, R., \& Palo, J. (1988). The Blessed Dementia Scale as a screening test for dementia. International Journal of Geriatric Psychiatry, 3, 267-273.

Gallagher, D., Rose, J., Rivera, P., Lovett, S., \& Thompson, L. W. (1989). Prevalence of depression in family caregivers. The Gerontologist, 29, $449-456$.

Gallagher, D., Wrabetz, A., Lovett, S., Del Maestro, S., \& Rose, J. (1989). Depression and other negative affects in family caregivers. In E. Light \& B. D. Lebowitz (Eds.), Alzheimer's disease treatment and family stress: Directions for research (pp. 218-244), Rockville, MD: National Institute of Mental Health.

Haley, W. E., Levine, E. G., Brown, S. L., Bartolucci, A. A. (1987). Stress, appraisal, coping, and social support as predictors of adaptational outcome among dementia caregivers. Psychology and Aging. 2, 323-330.

Hamilton, M. (1967). Development of a rating scale for primary depressive illness. British Journal of Social and Clinical Psychology, 6 , 278-296.

Kessler, R. C., Price, R. H., \& Wortman, C. B. (1985). Social factors in psychopathology: Stress, social support, and coping processes. $A n$ nual Review of Psychology, 36, 531-572.

Light, E., \& Lebowitz, B. D. (1989). Alzheimer's disease treatment and family stress: Directions for research. Rockville, MD: National Institute of Mental Health.

McKhann, G., Drachman, D., Folstein, M., Katzman, R., Price, D., \& Stadlan, E. (1984). Clinical diagnosis of Alzheimer's disease: Report of the NINCDS-ADRDA Work Group under the auspices of the Department of Health and Human Services Task Force on Alzheimer's Disease. Neurology, 34, 939-944.

Morris, R. G., Morris, L. W., \& Britton, P. G. (1988). Factors affecting the emotional well-being of the caregivers of dementia sufferers. British Journal of Psychiatry, 153, 147-156.

Popkin, M. K., Callies, A. L., Lentz, R. D., Colon, E. A., \& Sutherland, 
D. E. (1988). Prevalence of major depression, simple phobia, and other psychiatric disorders in patients with long-standing type I diabetes mellitus. Archives of General Psychiatry, 45, 64-68.

Quayhagen, M. P., \& Quayhagen, M. (1988). Alzheimer's stress: Coping with the caregiving role. The Gerontologist, 28, 391-396.

Reifler, B. V., Cox, G. B., \& Hanley, R. J. (1981). Problems of mentally ill elderly as perceived by patients, families, and clinicians. The Gerontologist, 21, 165-170.

Reisberg, B., Ferris, S. H., Deleon, M. J., \& Crook, T. (1982). The Global Deterioration Scale for assessment of primary degenerative dementia. American Journal of Psychiatry, 139, 1136-1139.

Riskind, J. H., Beck, A. T., Berchick, R. J., Brown, G., \& Steer, R. A. (1987). Reliability of DSM-III diagnoses for major depression and generalized anxiety disorder using the structured clinical interview for DSM-III. Archives of General Psychiatry, 44, 817-820.

Robinson, B., \& Thurnher, M. (1979). Taking care of aged parents: A family cycle transition. The Gerontologist, 19, 586-593.
Schulz, R., Tompkins, C. A., \& Rau, M. T. (1988). A longitudinal study of the psychosocial impact of stroke on primary support persons. Psychology and Aging, 3, 131-141.

Spitzer, R. L., Williams, J. B. W., Endicott, J., \& Gibbon, M. (1987). Structured Clinical Interview for DSM-III-R Disorders-Non-Patient version ( $S C I D-N P$ ). New York: New York State Psychiatric Institute, Biometrics Research Department.

Zarit, S., Reever, K., \& Bach-Peterson, J. (1980). Relatives of the impaired elderly: Correlates of feelings of burden. The Gerontologist, $20,649-655$.

Zimmerman, M., Coryell, W., Pfohl, B., \& Stangl, D. (1988). The reliability of the family history method of psychiatric diagnoses. Archives of General Psychiatry, 45, 320-322.

Received August 6, 1990

Revision received February 19, 1991

Accepted February 20, 1991

\title{
Call for Nominations for Neuropsychology
}

The APA Publications and Communications (P\&C) Board has opened nominations for the editorship of Neuropsychology for the years 1993-1998. B. P. Uzzell is the incumbent editor of this newly acquired APA journal in the area of experimental and applied neuropsychology, which will begin publication under APA in 1993.

Candidates must be members of APA and should be available to start receiving manuscripts in January 1992 to prepare for issues published in 1993. Please note that the P\&C Board encourages more participation by members of underrepresented groups in the publication process and would particularly welcome such nominees.

To nominate candidates, prepare a statement of one page or less in support of each candidate. Submit nominations to

\author{
Martha A. Storandt \\ Psychology Department \\ Washington University \\ 1 Brookings Drive \\ St. Louis, Missouri 63130
}

Other members of the search committee are Sandra P. Koffler, Charles G. Matthews, and Michael 1. Posner.

Nominations will be reviewed individually as received to ascertain nominees' interest in being considered. The search committee will begin systematic review of all nominations sometime after August 15,1991, and it is expected that a slate of possible nominees will be presented to the P\&C Board at its October 25-26, 1991, meeting. 